\title{
Percepciones estudiantiles acerca del uso de nuevas tecnologías en instituciones de Educación Superior en Medellín*
}

\author{
Salim Chalela Naffah**, Alejandro Valencia Arias***, \\ Jonathan Bermúdez Hernández ${ }^{* * *}$, Carlos Mario Ortega Rojas ${ }^{* * * * *}$
}

\begin{abstract}
Resumen
Introducción. La educación virtual ha crecido en los últimos años de manera exponencial en el ámbito mundial, y Colombia no ha sido la excepción. Objetivo. Describir las percepciones estudiantiles respecto de los factores que inciden en el proceso de aceptación de las herramientas virtuales de aprendizaje en la ciudad de Medellín. Materiales y métodos. Se desarrolló un diseño cuantitativo a través de la aplicación de cuestionarios autoadministrados a 1032 estudiantes universitarios. Resultados. La aparición de nuevas tecnologías de la información y las comunicaciones, el incremento de la velocidad en los tiempos de transacciones e intercambios, entre otras condiciones, han incidido en las estrategias de masificación y flexibilización para la formación en algunas instituciones de Educación Superior. Conclusión. Se concluyó que, aunque las plataformas virtuales permiten a los estudiantes sentirse más eficaces en su proceso de aprendizaje, aún existen grandes retos para la implementación de nuevas tecnologías como estrategias de mediación del proceso enseñanzaaprendizaje.
\end{abstract}

Palabras clave: aprendizaje virtual; educación superior; percepción; adaptación tecnológica; relación estudiante-profesor.
Perceptions on the use of new technologies among highe education students in Medellin

\begin{abstract}
Introduction. Online education has grown exponentially in recent years worldwide, and Colombia has not been an exception. Objective. Describe the perceptions of students about the factors that affect the acceptance of online learning tools in Medellín. Materials and methods. A quantitative design by the use of self-managed questionnaires for 1032 students was used. Results. New information and communications technologies, faster transactions and exchanges and many other conditions have affected massification and flexibility strategies in some higher education institutions. Conclusions. The conclusion was that, even though online platforms help students feel more efficient in their learning processes, there are still many challenges to be faced concerning the implementation of new technologies as strategies to mediate in the teaching-learning process.
\end{abstract}

Key words: online learning, higher education, perception; technological adaptation; studentteacher relationship.

\footnotetext{
* $\quad$ El artículo es resultado del proyecto de investigación titulado "Evaluación del uso y la aceptación de las herramientas virtuales de aprendizaje estudiantes universitarios de la ciudad de Medellín a través del Modelo de Aceptación Tecnológica (TAM)", financiado por la Universidad Autónoma Latinoamericana - Unaula-, el Instituto Tecnológico Metropolitano -ITM-, y la Institución Universitaria Escolme. Código: 17-000022.

** Coordinador de investigaciones en la Escuela de Posgrados Universidad Autónoma Latinoamericana. Estudiante Doctorado en Educación. Universidad Autónoma de Barcelona. E-mail. salim.chalela@unaula.edu.co

*** Docente en el Instituto Tecnológico Metropolitano. Estudiante Doctorado en Ingeniería, Industria y Organizaciones de la Universidad Nacional de Colombia. E- mail. jhoanyvalencia@itm.edu.co

**** Docente en el Instituto Tecnológico Metropolitano. Magíster en Administración de la Universidad Nacional de Colombia. E-mail. jonathanbermudez@itm.edu.co

**** Coordinador de Investigaciones, Institución Universitaria Escolme. Magíster en Educación de la Universidad de Medellín. E-mail. cies@escolme.edu.co
}

Autor para correspondencia: Salim Chalela Naffah, email: salim.chalela@unaula.edu.co Artículo recibido: 19/02/2016; Artículo aprobado: 15/11/2016 


\section{Percepções estudantis sobre o uso de novas tecnologias em instituições de educação universitária em Medellín}

\section{Resumo}

Introdução. A educação virtual há crescido nos últimos anos de maneira exponencial no âmbito mundial, e a Colômbia não há sido a exceção. Objetivo. Identificar as percepções estudantis com respeito aos fatores que incidem no processo de aceitação das ferramentas virtuais de aprendizagem na cidade de Medellín. Materiais e métodos. Se desenvolveu um desenho quantitativo através da aplicação de questionários auto-administrados a 1032 estudantes universitários. Resultados.
A aparição de novas tecnologias da informação e as comunicações, o incremento da velocidade nos tempos de transações e intercâmbios, entre outras condições, há incidido nas estratégias de massificação e flexibilização para a formação em algumas instituições de Educação Universitária. Conclusões. Se concluiu que, ainda que as plataformas virtuais permitem aos estudantes sentir-se mais eficazes no seu processo de aprendizagem, ainda existem grandes desafios para a implementação de novas tecnologias como estratégias de mediação do processo ensinoaprendizagem.

Palavras chave: aprendizagem virtual; educação universitária; percepção; adaptação tecnológica; relação estudante-professor.

\section{Introducción}

La virtualidad es, sin duda, una de las principales nociones utilizadas para describir los entornos de interacción que han surgido en la contemporaneidad por cuenta de los avances en el uso y apropiación de las nuevas tecnologías de la información y las comunicaciones -TIC-, como la Internet (Fragoso y González, 2013). Uno de los campos en los que ha tenido un alto nivel la utilización de estas nuevas tecnologías es la educación, dando origen a lo que se conoce como educación virtual (Mohammadyari y Singh, 2015; Paechter y Maier, 2010).

La educación virtual, es decir, desde la nopresencia física en un espacio geográfico determinado, no se sitúa necesariamente en ninguna orientación pedagógica concreta. Al igual que en las herramientas tradicionales, el acto educativo se nutre de la armonía entre pedagogía y didáctica, al que se suma una mayor autonomía del estudiante (Artino, 2010; Sánchez y Hueros, 2010). Por tanto, el aprendizaje virtual (e-learning) es el resultado de la interacción entre el docente y el estudiante, mediado por las herramientas tecnológicas que se utilizan en el proceso de enseñanza (Ozdamli y Uzunboylu, 2015; Artino y Jones, 2012; Chen, 2011), en el que la potencialidad e incidencia están dadas por la capacidad de los individuos de aceptación y adaptación al uso de las nuevas tecnologías de la información y las comunicaciones (Hernández-Ramos, Martínez-Abad, Peñalvo, García y RodríguezConde, 2014; McGill, Klobas y Renzi, 2014).

Respecto a la capacidad de adaptación de las tecnologías a los procesos educativos, se ha mencionado que esta condición está relacionada con las plataformas virtuales que se han desarrollado para facilitar la interacción entre los individuos que participan del proceso enseñanza-aprendizaje (Benta, Bologa y Dzitac, 2014). Existen múltiples herramientas virtuales como: los portales de distribución de contenidos, los entornos de trabajo en grupo o colaboración, los sistemas de gestión de contenidos (CMS), los sistemas de gestión de conocimiento (LMS), los sistemas de gestión de contenidos para el conocimiento o aprendizaje (LCMS), y los objetos virtuales de aprendizaje (OVA), entre otros. Vale la pena resaltar que el tipo de entorno adecuado para la educación virtual son los sistemas de gestión del conocimiento (LMS), también denominados Entornos Virtuales de Aprendizaje (EVA); sin embargo, lo básico o avanzado en la adecuación de estos sistemas requiere de un análisis de las capacidades institucionales para el desarrollo de su infraestructura, así como de la usabilidad y los niveles de aceptación tecnológica que los individuos tengan de estos. (Essalmi, Ayed, Jemni y Graf, 2010). 
Frente al uso de estas herramientas virtuales, en múltiples investigaciones se han exaltado las ventajas que ofrecen respecto a los procesos de formación tradicional, como: bajos costos para acceder a programas académicos (Lee y Hung, 2015); flexibilidad horaria para realizar las actividades propuestas en cada programa (Tarhini, Hassouna, Abbasi y Orozco, 2015); ahorro en tiempo; facilidad de acceso a información por múltiples vías (visual, auditiva, interactiva); innovación en la pedagogía y la didáctica, entre otros (McGill et al., 2014). También se han encontrado ventajas respecto de la actitud de los individuos para utilizar las tecnologías de la información y las comunicaciones como herramientas para mediar el aprendizaje: la autonomía de la que disponen para avanzar en su proceso formativo; el fortalecimiento de habilidades técnicas en uso de tecnologías de la información y las comunicaciones como "valor agregado" al aprendizaje de su campo disciplinar de estudio; la capacidad de este tipo de herramientas de adaptarse a los diferentes estilos de aprendizaje que tienen los individuos; los cambios en las percepciones que tienen acerca de las finalidades de las tareas o los talleres evaluativos, entre otras (El-Seoud, Taj-Eddin, Seddiek, El-Khouly y Nosseir, 2014; Benta et al., 2014; Klašnja-Milićević, Vesin, Ivanović y Budimac, 2011).

El aprendizaje virtual o e-learning ha evolucionado de manera significativa, y se ha situado en la convergencia de una serie de factores individuales, tecnológicos e institucionales, que alimentan los recursos y contenidos de interacción, en cualquiera de las herramientas virtuales que se utilice, para su adecuada aceptación (Hamidi, Meshkat, Rezaee y Jafari, 2011). El diseño de la interfaz y del soporte, en función de las dinámicas administrativas y académicas propias de cualquier institución educativa, se convierte en la columna vertebral de un sistema adecuado y comprensible, tanto para la institución como para los individuos que acceden a él (Garrison, 2011; Rosenberg, 2001). Sin embargo, la discusión contemporánea que surge en función del rol que juegan tanto la aceptación tecnológica como el proceso educativo, en el avance de la implementación de la educación, centrado en el aprendizaje virtual, ha generado tensiones y debates acerca de estas nuevas formas de aprendizaje mediadas por la virtualidad (Domingo y Garganté, 2016; González, García y Ramírez, 2015; Zhang, Wang, de Pablos, Tang y Yan, 2015). Según la Unesco, estas expresiones son limitadas ante el desarrollo de la cultura digital del nuevo siglo, que se organiza como una sociedad, o más bien sociedades de la información y el conocimiento (Unesco, 2005). Por tanto, existen condiciones que son consideradas como debilidades a la hora de implementar este tipo de herramientas, en razón a que ponen en riesgo la eficiencia y la efectividad de las plataformas virtuales para alcanzar los objetivos formativos esperados. Algunas de ellas son: el analfabetismo y la brecha digital; la despersonalización de la relación docente; la falta de confianza y experiencia en el uso de las herramientas virtuales para aprender; la incapacidad del docente de acoplar sus estrategias pedagógicas a las nuevas tecnologías de la información y las comunicaciones, y las emociones que produce en el estudiante el aislamiento por la excesiva autonomía del proceso formativo que han sido significativas en múltiples investigaciones (Benta et al., 2014; Artino y Jones, 2012; Lan y Sie, 2010; Norris, 2001).

\section{Metodología}

Con el objetivo de describir las percepciones que tienen los estudiantes universitarios con relación al uso y la utilidad de las herramientas virtuales en el proceso de aprendizaje, se propuso un análisis fundamentado en métodos de recolección de información cuantitativos, de las variables determinantes del modelo de aceptación tecnológica -TAM- (Mohammadi, 2015; Paechter y Maier, 2010).

El modelo de aceptación tecnológica -TAMfue escogido para esta investigación en razón a que centra su atención en las actitudes e intenciones del individuo respecto a la utilidad y la facilidad de uso de las tecnologías de la información y las comunicaciones como mediadores del proceso de enseñanzaaprendizaje, por lo que se ha convertido en uno de los modelos más utilizados para analizar la percepción de docentes y estudiantes 
respecto del impacto que han tenido las TIC en las estrategias de e-learning en diferentes niveles de formación educativa (Mohammadi, 2015; Tarhini et al.,2015; Lu, 2012; Chen, 2011; Sánchez y Hueros, 2010).

Las variables determinantes del modelo de aceptación tecnológica son la facilidad de uso (FU), la percepción de utilidad (UP), la actitud frente al uso (ACT) y la intención de uso (INT) (Paechter y Maier, 2010; Park, 2009). Es importante considerar que las primeras dos variables están condicionadas por las percepciones, inherentes o externas, que tienen los individuos acerca de las plataformas virtuales. Por tanto, teniendo como referencia algunas investigaciones en la actualidad, se consideró que algunas percepciones de los estudiantes respecto a la autonomía en el aprendizaje (AA), la auto-eficiencia percibida (PA), la preparación del estudiante (Pest), la disposición a innovar -Personal Innovativeness (PI)- y la percepción que tienen respecto de las habilidades de los docentes para utilizar las plataformas virtuales como herramientas de e-learning (preparación del instructor (Pins)) eran relevantes para la presente investigación
(Park y Lim, 2015; El-Seoud et al., 2014; Artino y Jones, 2012; Lu, 2012).

Definidas las variables, se construyó un cuestionario que contenía dieciocho preguntas asociadas a las mismas. La escala de medida utilizada fue la Likert con cinco opciones de respuesta (1. Muy en desacuerdo - 5. Muy de acuerdo) (tablas 1 y 2). Además, se generó una batería de preguntas asociadas a condiciones socio-demográficas de los estudiantes como edad, institución de Educación Superior a la que están vinculados, tipo de formación (técnica, tecnológica o profesional), programa académico que están estudiando. En ese mismo orden de ideas, se les consultó por el interés, conocimiento y periodicidad de ingreso a las plataformas virtuales de sus instituciones universitarias.

Dicho cuestionario fue auto-administrado a 1032 estudiantes vinculados a programas de pregrado en diferentes disciplinas de las ciencias exactas y las ciencias humanas y sociales de tres instituciones de Educación Superior de la ciudad de Medellín, entre los meses de abril y junio del año 2015.

Tabla 1. Número de constructos asociados a cada una de las variables

\begin{tabular}{c|c|c}
\hline $\begin{array}{c}\text { Identificación de } \\
\text { la variable }\end{array}$ & Variable & $\begin{array}{c}\text { Número de } \\
\text { preguntas }\end{array}$ \\
\hline FU & Facilidad de uso & 2 \\
\hline PU & Percepción de utilidad & 3 \\
\hline ACT & Actitud & 2 \\
\hline INT & Intención & 1 \\
\hline PIns & Preparación del Instructor & 3 \\
\hline AA & Autonomía en el aprendizaje & 2 \\
\hline PA & Auto-eficiencia percibida & 2 \\
\hline Pest & Preparación del estudiante & 2 \\
\hline PI & Personal Innovativeness & 2 \\
\hline
\end{tabular}

Fuente: elaboración propia

La recolección de la información fue procesada y sistematizada usando el "Statistical Product and Service Solution"-SPSS-; posteriormente, cada una de las variables fue sometida a un test de confiabilidad estadística, utilizando el Alpha de Cronbrach. En la tabla 3, se presentan los resultados obtenidos en la prueba de confiabilidad. Como puede apreciarse, los valores obtenidos para cada variable son superiores a 0.70 , lo que confirma su confiabilidad y permite analizar sus resultados (Oviedo y Campo-Arias, 2005). 
Tabla 2. Descripción de constructos asociados a cada una de las variables

\begin{tabular}{|c|c|}
\hline $\begin{array}{l}\text { Identificación } \\
\text { de la variable }\end{array}$ & Constructos \\
\hline FU & $\begin{array}{l}\text { FU1. Considero fácil acceder a los contenidos virtuales de la plataforma virtual } \\
\text { FU2. No tengo ninguna dificultad usando las herramientas de aprendizaje de la plataforma } \\
\text { virtual }\end{array}$ \\
\hline PU & $\begin{array}{l}\text { PU1. La utilización de la plataforma virtual me permite realizar mis trabajos de forma más } \\
\text { rápida } \\
\text { PU2. Creo que las herramientas que incorpora la plataforma virtual (cuestionarios, chat, } \\
\text { foros) son útiles para mi aprendizaje } \\
\text { PU3. Considero que tendría más oportunidades de adquirir conocimientos en la plataforma } \\
\text { virtual si se utilizarán más herramientas virtuales de aprendizaje }\end{array}$ \\
\hline ACT & $\begin{array}{l}\text { ACT1. Disfrutaría más el trabajo de las materias si se incorporara un mayor número de } \\
\text { herramientas de aprendizaje en la plataforma virtual } \\
\text { ACT2. Me gusta acceder a las herramientas de la plataforma virtual }\end{array}$ \\
\hline INT & $\begin{array}{l}\text { INT1. Tengo la intención de dar un mayor uso a la plataforma virtual para mejorar mis } \\
\text { procesos de aprendizaje }\end{array}$ \\
\hline PIns & $\begin{array}{l}\text { PIns1. Los docentes me motivan por utilizar las tecnologías de información y comunicación } \\
\text { (TIC) en mis procesos de aprendizaje } \\
\text { PIns2. Pienso que los docentes deberían dar un mayor uso a la plataforma virtual en los } \\
\text { procesos de enseñanza } \\
\text { PIns3. Los docentes tienen los conocimientos adecuados para la utilización de las TIC en } \\
\text { sus asignaturas }\end{array}$ \\
\hline AA & $\begin{array}{l}\text { AA1. Creo que usar la plataforma virtual en las asignaturas mejora mis habilidades para } \\
\text { el aprendizaje } \\
\text { AA2. Me gusta ser autónomo en mi ritmo de aprendizaje }\end{array}$ \\
\hline PA & $\begin{array}{l}\text { PA1. Tengo las habilidades necesarias para mejorar mi aprendizaje con ayuda de la pla- } \\
\text { taforma virtual } \\
\text { PA2. Tengo los conocimientos necesarios para utilizar las herramientas virtuales de apren- } \\
\text { dizaje disponibles en la plataforma virtual }\end{array}$ \\
\hline Pest & $\begin{array}{l}\text { Pest1. Percibo que mis compañeros valoran la plataforma virtual como una herramienta } \\
\text { útil en el programa académico } \\
\text { Pest2. Los estudiantes deberíamos recibir mayores capacitaciones para una adecuada } \\
\text { utilización de la plataforma virtual en nuestras asignaturas }\end{array}$ \\
\hline PI & $\begin{array}{l}\text { PI1. Me gusta conocer sobre nuevas tecnologías de la información y la comunicación (TIC) } \\
\text { PI2. Entre mis compañeros, soy de los primeros en probar nuevas herramientas tecnoló- } \\
\text { gicas }\end{array}$ \\
\hline
\end{tabular}

Tabla 3. Prueba de confiabilidad de variables

\begin{tabular}{c|c}
\hline Identificación de la variable & Alfa de Cronbrach \\
\hline FU & 0.909 \\
\hline PU & 0.725 \\
\hline ACT & 0.878 \\
\hline PIns & 0.750 \\
\hline AA & 0.843 \\
\hline PA & 0.885 \\
\hline Pest & 0.875 \\
\hline PI & 0.830 \\
\hline
\end{tabular}

Fuente: elaboración propia 


\section{Resultados y discusión}

Para analizar las percepciones que tienen los estudiantes respecto de la facilidad de uso y la utilidad de las herramientas de e-learning para la mediación del proceso de enseñanzaaprendizaje en la Educación Superior es importante considerar, inicialmente, los rasgos socio-demográficos que caracterizan la muestra, así como el interés, el nivel de conocimiento y la periodicidad en el acceso a las plataformas tecnológicas que las instituciones universitarias han diseñado para la interacción virtual.

Las tres instituciones de educación superior seleccionadas para la investigación (Instituto Tecnológico Metropolitano -ITM-, Universidad Autónoma Latinoamericana -Unaula- e Institución Universitaria Escolme) acogen estudiantes de estrato socioeconómico medio bajo (uno, dos y tres), en los que el precio de la matrícula oscila entre tres y cinco salarios mínimos legales vigentes ${ }^{1}$. La edad promedio de los estudiantes es de 25 años; 44,05 \% son mujeres y $55,95 \%$, hombres. Además, el $69,39 \%$ se encuentra cursando programas académicos relacionados con las ciencias administrativas, económicas y contables; el $12,05 \%$, programas en los campos de las ciencias sociales y humanas; y el 18,56 $\%$, disciplinas de la ingeniería. Finalmente, $36,05 \%$ están matriculados en programas profesionales, mientras que $63,95 \%$, en programas de nivel técnico y tecnológico.

En cuanto al interés por utilizar las plataformas virtuales, el 57,64 \% de los estudiantes manifestó que le gusta que se utilicen plataformas virtuales como herramientas de mediación e interacción con los docentes, mientras que a un $41,28 \%$ no les gusta. De lo anterior, se puede observar que aún existe un alto porcentaje de estudiantes universitarios que, a pesar de estar familiarizados con el uso de dispositivos digitales en la cotidianidad, no les interesa utilizar las plataformas virtuales como herramientas de mediación pedagógica en el aula, lo que concuerda con los hallazgos de Domingo y Garganté (2016) y El-Seoud, et al. ( 2014).
En consideración al nivel de conocimiento frente al funcionamiento y el uso de las plataformas virtuales habilitadas por las tres instituciones, 53,63 \% señalaron tener un nivel medio, mientras que $24,17 \%$ dijeron no saber utilizar este tipo de herramientas. Vale la pena resaltar que solo $16,18 \%$ indicaron tener un buen conocimiento de las herramientas, un porcentaje muy bajo que se convierte en factor crítico para incorporar este tipo de estrategias pedagógicas en los currículos universitarios como lo plantean McGill et al., (2014).

Finalmente, el 84,98\% de los estudiantes utiliza las plataformas virtuales de las instituciones de Educación Superior en las que se encuentran matriculados. Frente a esto, el 37,05 \% ingresa entre una y dos veces por semana; el 19,58 \% lo hace entre tres y cuatro veces por semana; el $8,21 \%$, entre cinco y seis veces por semanas, y tan solo $10,42 \%$ ingresa más de seis veces, por lo que se infiere que la mayoría de los estudiantes accede a este tipo de herramientas solamente ante requerimientos específicos en algún curso, y no es un usuario que interactúa de manera activa en estos escenarios, coincidiendo con los hallazgos expuestos por González et al. (2015).

Definido el perfil socio-demográfico de los estudiantes, y su interés respecto del funcionamiento de las herramientas para el aprendizaje virtual, a continuación, se presenta un análisis descriptivo de sus percepciones con relación a la facilidad de uso, utilidad, actitud e intención, condiciones determinantes del modelo de aceptación tecnológica utilizado en la presente investigación.

En primer lugar, en cuanto a la facilidad de uso que perciben los estudiantes de las plataformas virtuales, la mayoría de los estudiantes está de acuerdo o muy de acuerdo $(70,43 \%)$ en que el acceso a los contenidos que ofrecen las plataformas es fácil; en consecuencia, no tienen dificultades para acceder y usar la plataforma $61.22 \%$ (tabla 4). Los resultados obtenidos dan cuenta de que cada día se avanza de manera significativa en generar objetos virtuales de aprendizaje intuitivos y

1 Como referencia, el Salario Mínimo Mensual Legal Vigente -SMMLV-en Colombia para el año 2016 es de $\$ 689.455$ pesos colombianos. 
versátiles, que les permiten a los estudiantes encontrar rápidamente la información, acceder de manera directa a diferentes canales de información y motores de búsqueda textual o audiovisual, compartir contenidos, documentos y otro tipo de archivos, interactuar y desarrollar actividades en tiempo real en grupos de trabajo, entre otras funciones que optimizan el proceso de enseñanza (González et al., 2015).

En cuanto a la percepción de utilidad que tienen los estudiantes respecto de las plataformas virtuales para el aprendizaje, si bien están de acuerdo con que los sistemas de e-learning les permiten desarrollar de manera más eficiente sus compromisos académicos $(36,35$
$\%$, y los diferentes espacios de interacción que se habilitan, como foros, salas de chat, cuestionarios, entre otros, son de utilidad para el aprendizaje (42,66 \%), más de un $64 \%$ está acuerdo o muy de acuerdo con que se podrían aprovechar más las plataformas virtuales para el e-learning, por lo que reconocen el potencial de las herramientas virtuales para fortalecer sus procesos de aprendizaje, lo cual se convierte en una ventaja al momento de formular estrategias para impulsar su uso, y garantiza mejores procesos de adopción de nuevas tecnologías, así como mayor apertura hacia nuevas opciones pedagógicas asociadas al uso de TIC (tabla 4).

Tabla 4. Facilidad de uso y percepción de utilidad

\begin{tabular}{c|c|c|c|c|c|c}
\hline Variable & $\begin{array}{c}\text { Muy de } \\
\text { acuerdo }\end{array}$ & $\begin{array}{c}\text { De } \\
\text { acuerdo }\end{array}$ & $\begin{array}{c}\text { Ni en acuerdo, ni } \\
\text { en desacuerdo }\end{array}$ & $\begin{array}{c}\text { En } \\
\text { desacuerdo }\end{array}$ & $\begin{array}{c}\text { Muy en } \\
\text { desacuerdo }\end{array}$ & Ns/Nr \\
\hline $\begin{array}{c}\text { Facilidad de uso } \\
\text { FU1 }\end{array}$ & $27.80 \%$ & $42.63 \%$ & $13.07 \%$ & $7.88 \%$ & $2.70 \%$ & $5.91 \%$ \\
\hline FU2 & $21.93 \%$ & $39.29 \%$ & $17.57 \%$ & $12.68 \%$ & $3.43 \%$ & $5.09 \%$ \\
\hline $\begin{array}{c}\text { Percepción de } \\
\text { utilidad PU1 }\end{array}$ & $18.75 \%$ & $36.35 \%$ & $24.69 \%$ & $9.58 \%$ & $3.85 \%$ & $6.77 \%$ \\
\hline PU2 & $19.77 \%$ & $42.66 \%$ & $19.35 \%$ & $8.22 \%$ & $3.95 \%$ & $6.04 \%$ \\
\hline PU3 & $24.53 \%$ & $40.84 \%$ & $20.53 \%$ & $6.63 \%$ & $3.26 \%$ & $4.21 \%$ \\
\hline
\end{tabular}

Fuente: elaboración propias

Aunque la información anteriormente expuesta es relevante, tanto las percepciones de facilidad de uso como de utilidad están condicionadas por dinámicas psicológicas de los individuos, tales como la actitud (ACT) y la intención (INT) respecto de las nuevas tecnologías como mediadoras del proceso de enseñanza-aprendizaje, así como de sus relaciones con los docentes. Adicionalmente, como se mencionó en el proceso metodológico, diferentes investigaciones han encontrado que las percepciones que tienen los estudiantes respecto a la autonomía en el aprendizaje (AA), la auto-eficiencia (PA), la preparación del estudiante (Pest), la auto-innovación (PI) y la preparación del instructor (Pins) también son determinantes en el proceso de adaptación de las tecnologías al acto educativo como lo han descrito Hung et al. (2016); Park y Lim (2015); González et al. (2015); Artino y Jones (2012) y Lu (2012).
En la tabla 5 se presentan los resultados del análisis de las variables Actitud (ACT) e Intención (INT), latentes en la teoría de la acción razonada, y determinantes tanto en el comportamiento como en las creencias de los individuos con relación a los modelos de aceptación tecnológica. Frente a la actitud, el $63,90 \%$ de los estudiantes mencionaron estar entre "muy de acuerdo" y "de acuerdo" en que disfrutarían más el trabajo de las materias si se incorporara un mayor número de herramientas de aprendizaje en la plataforma virtual. Además, al $55,9 \%$ le gusta acceder a las herramientas de la plataforma virtual.

Por otro lado, el $64,8 \%$ de los encuestados estuvieron de acuerdo con tener la intención de dar un mayor uso a la plataforma virtual para mejorar sus procesos de aprendizaje; aunque es un porcentaje significativo, el 20,6 $\%$ se mostró indiferente frente a esta intención 
y el 9,4\% no manifestó no tener la intención de hacerlo, por lo que se evidencia que, a pesar de utilizar las plataformas virtuales, aún hay un número significativo de estudiantes que prefiere utilizar mecanismos tradicionales para acceder a los contenidos temáticos en su proceso de formación, como lo encontraron Paechter y Maier (2010). Por lo tanto, es indispensable generar estrategias didácticas y pedagógicas que permitan apropiar las herramientas tecnológicas en esta parte de la población que aún se muestra escéptica frente a las potencialidades de las plataformas virtuales para el fortalecimiento de los procesos de enseñanza y aprendizaje.

Tabla 5. Actitud e intención de los estudiantes respecto al uso de plataformas virtuales

\begin{tabular}{|c|c|c|c|c|c|c|}
\hline Variable & $\begin{array}{l}\text { Muy de } \\
\text { acuerdo }\end{array}$ & $\begin{array}{c}\text { De } \\
\text { acuerdo }\end{array}$ & $\begin{array}{l}\text { Ni en acuerdo, ni } \\
\text { en desacuerdo }\end{array}$ & $\begin{array}{c}\text { En } \\
\text { desacuerdo }\end{array}$ & $\begin{array}{c}\text { Muy en } \\
\text { desacuerdo }\end{array}$ & $\mathrm{Ns} / \mathrm{Nr}$ \\
\hline $\begin{array}{l}\text { Actitud } \\
\text { ACT1 } \\
\text { ACT2 } \\
\end{array}$ & $\begin{array}{l}27.39 \% \\
16.25 \%\end{array}$ & $\begin{array}{l}36.51 \% \\
39.58 \%\end{array}$ & $\begin{array}{l}18.05 \% \\
23.02 \%\end{array}$ & $\begin{array}{l}10.37 \% \\
10.73 \%\end{array}$ & $\begin{array}{l}3.63 \% \\
4.79 \%\end{array}$ & $\begin{array}{l}4.05 \% \\
5.63 \%\end{array}$ \\
\hline $\begin{array}{c}\text { Intención } \\
\text { INT1 }\end{array}$ & $20.97 \%$ & $43.84 \%$ & $20.65 \%$ & $7.27 \%$ & $3.16 \%$ & $4.11 \%$ \\
\hline
\end{tabular}

Fuente: elaboración propia

La tabla 6 presenta los resultados de la percepción que tienen los estudiantes respecto a sus aptitudes para la utilización de plataformas virtuales (preparación, autonomía, disposición para la innovación -Personal Innovativeness- y auto-eficiencia para el aprendizaje). También sus percepciones frente a las capacidades de los docentes para la utilización de las herramientas virtuales como estrategias pedagógicas. Los resultados obtenidos del análisis de variables externas del TAM reflejan hallazgos interesantes, considerando la variedad de constructos propuestos paras las cinco variables analizadas.

En primer lugar, es clara la tendencia simétrica en la distribución de frecuencias de las variables que corresponden a la percepción positiva que tiene el estudiante acerca de las cualidades que lo habilitarían para utilizar las plataformas virtuales como herramienta de mediación en el proceso de enseñanza-aprendizaje, como lo son su preparación, su grado de autonomía para el aprendizaje, la auto-eficiencia y su disposición para la innovación en el uso de este tipo de herramientas. Esto contrasta con el hecho de que la percepción del estudiante respecto a la preparación del instructor, si bien es positiva, tiene una tendencia hacia la neutralidad.

Al analizar los tres constructos que componen la percepción acerca de la preparación del instructor, se encontró que la tendencia hacia la neutralidad está dada por que los estudiantes no perciben claramente que los docentes los motiven a utilizar las plataformas virtuales para mediar el aprendizaje (22,96\%); ponen en duda los conocimientos de los docentes de las plataformas virtuales $(28,04 \%)$, y están de acuerdo en que los docentes deberían dar un mayor uso de estas plataformas para la enseñanza $(38,84 \%)$. Es decir que, como lo encontraron Hung et al. (2016), la falta de una percepción positiva de los estudiantes respecto de la preparación de los docentes en el uso de herramientas virtuales podría incidir en sus actitudes e intenciones para utilizar con mayor frecuencia las plataformas virtuales de las que disponen.

En segundo lugar, los estudiantes están de acuerdo en que el aprendizaje mediado por la virtualidad les posibilita controlar sus ritmos y, por ende, ser autónomos en su proceso. Este comportamiento se refuerza con los resultados obtenidos al preguntárles acerca de la percepción que tienen de la utilidad que les dan sus compañeros de clase a las plataformas virtuales, en los que se evidencia que hay una valoración neutral (ni positiva, ni negativa) respecto a la utilidad que como colectivo tienen de estas herramientas para el aprendizaje. En este apartado, es importante resaltar que los 
estudiantes, en su gran mayoría, están muy de acuerdo o de acuerdo con que las instituciones deben ofrecerles capacitaciones acerca del e-learning, su pertinencia y utilidad, y no solamente quedarse en la operación técnica de las plataformas disponibles.

Además, es importante resaltar que entre los encuestados existe indiferencia, es decir, que no se sienten interesados por ser los primeros en probar nuevas herramientas tecnológicas, como lo afirmó el 33,9\%, lo que puede conllevar dificultades al momento de incorporar nuevas herramientas. Por otro lado, el $35,4 \%$ percibe que sus compañeros valoran la plataforma virtual como una herramienta útil en sus carreras; por lo tanto, aún se observa una subvaloración de las potencialidades de las plataformas virtuales como herramientas de enseñanza y aprendizaje. Esto se reafirma con el hecho de que el $62,7 \%$ de los encuestados mencionó estar de acuerdo con tener conocimientos necesarios para utilizar las herramientas virtuales de aprendizaje disponibles en la plataforma virtual; sin embargo, existe aún un porcentaje importante de estudiantes a los cuales se debe capacitar y formar para el uso adecuado de las plataformas.

Tabla 6. Percepción de los estudiantes acerca de sus habilidades y las de los docentes para potenciar el aprendizaje virtual

\begin{tabular}{c|c|c|c|c|c|c}
\hline Variable & $\begin{array}{c}\text { Muy de } \\
\text { acuerdo }\end{array}$ & $\begin{array}{c}\text { De } \\
\text { acuerdo }\end{array}$ & $\begin{array}{c}\text { Ni en acuerdo, ni } \\
\text { en desacuerdo }\end{array}$ & En desacuerdo & $\begin{array}{c}\text { Muy en des- } \\
\text { acuerdo }\end{array}$ & Ns/Nr \\
\hline $\begin{array}{c}\text { Preparación del e } \\
\text { studiante } \\
\text { Pest1 }\end{array}$ & $10.65 \%$ & $24.84 \%$ & $30.48 \%$ & $19.31 \%$ & $8.04 \%$ & $6.68 \%$ \\
\hline Pest2 & $36.34 \%$ & $39.71 \%$ & $4.94 \%$ & $4.94 \%$ & $2.10 \%$ & $2.94 \%$ \\
\hline $\begin{array}{c}\text { Autonomía } \\
\text { AA1 }\end{array}$ & $15.80 \%$ & $38.67 \%$ & $23.18 \%$ & $12.89 \%$ & $3.74 \%$ & $5.72 \%$ \\
\hline AA2 & $33.12 \%$ & $44.31 \%$ & $12.33 \%$ & $5.43 \%$ & $1.67 \%$ & $3.13 \%$ \\
\hline $\begin{array}{c}\text { Auto-eficiencia } \\
\text { percibida } \\
\text { PA1 }\end{array}$ & $18.68 \%$ & $44.99 \%$ & $20.35 \%$ & $8.04 \%$ & $2.92 \%$ & $5.01 \%$ \\
\hline PA2 & $21.99 \%$ & $41.75 \%$ & $17.34 \%$ & $11.21 \%$ & $3.38 \%$ & $4.33 \%$ \\
\hline $\begin{array}{c}\text { Personal } \\
\text { Innovativeness } \\
\text { PI1 }\end{array}$ & $35.55 \%$ & $41.79 \%$ & $14.03 \%$ & $3.95 \%$ & $2.18 \%$ & $2.49 \%$ \\
\hline PI2 & $12.75 \%$ & $25.72 \%$ & $33.90 \%$ & $13.82 \%$ & $4.99 \%$ & $8.82 \%$ \\
\hline $\begin{array}{c}\text { Preparación del } \\
\text { Instructor } \\
\text { PIns1 }\end{array}$ & $15.55 \%$ & $28.81 \%$ & $22.96 \%$ & $18.48 \%$ & $10.33 \%$ & $3.86 \%$ \\
\hline PIns2 & $28.32 \%$ & $38.84 \%$ & $18.00 \%$ & $8.84 \%$ & $2.74 \%$ & $3.26 \%$ \\
\hline PIns3 & $15.34 \%$ & $32.59 \%$ & $28.04 \%$ & $11.96 \%$ & $5.19 \%$ & $6.88 \%$ \\
\hline
\end{tabular}

Fuente: elaboración propia

\section{Conclusiones}

La educación virtual ha crecido en los últimos años de manera exponencial en el mundo, y Colombia no ha sido la excepción. La aparición de nuevas tecnologías de la información y las comunicaciones, el incremento de la velocidad en los tiempos de transacciones e intercambios, la gestión del conocimiento, entre otras condiciones, han incidido para que las instituciones de Educación superior promuevan estrategias de masificación, flexibilización y modernización de un proceso formativo.

En este orden de ideas, las herramientas virtuales de enseñanza-aprendizaje, en las instituciones de Educación Superior, no solamente están influidas en alto grado por el nivel de competencias informáticas que 
posean los estudiantes universitarios, sino que también es importante considerar la percepción que ellos tienen respecto a la utilidad de las mismas como herramienta de mediación pedagógica, la facilidad de uso con que los estudiantes perciben las herramientas, la actitud de su círculo social y de ellos hacia dichas herramientas, elementos que se ven reflejados en la intención que tendrán de utilizarlas. Frente a esto, aunque se observaron buenos resultados, aparecen retos para fortalecer la utilidad del e-learning respecto de herramientas tradicionales permitiendo que identifiquen de forma más directa las ventajas del uso de herramientas virtuales de aprendizaje para mejorar los procesos de enseñanza y aprendizaje.

Los estudiantes encuestados consideran que las herramientas que incorpora la plataforma (cuestionarios, chats, foros) son útiles a la hora de enriquecer el conocimiento y contribuyen a la mejora continua del aprendizaje, y perciben que tendrían más oportunidades de adquirir conocimientos en la plataforma si se utilizaran más herramientas virtuales de aprendizaje. Lo anterior muestra que la población estudiantil manifiesta gran interés en recibir una formación que integre el aprendizaje de instrumentos informáticos como una competencia profesional con el mismo valor de otros saberes que se aprenden de forma regular, resultados que son coherentes con lo planteado por McGill et al. (2014), en su investigación sobre factores críticos influyentes en la utilización del e-learning.

Con relación a la utilidad de las herramientas virtuales, se puede concluir que estas, como instrumento de apoyo para la realización de trabajos y actividades curriculares y extracurriculares, permiten a los estudiantes sentirse más eficaces en cuanto al cumplimiento de las actividades propuestas al nivel individual, reafirmando lo encontrado en la investigación de Park y Lim (2015); sin embargo, algunas investigaciones han encontrado que esto puede generar algunas complicaciones respecto al trabajo colectivo por cuanto los niveles y ritmos de aprendizaje pueden no sincronizarse (González, et al., 2015), por lo que es necesario que las instituciones de Educación Superior no se limiten a verificar que el estudiante posea competencias informáticas, sino que procuren por el desarrollo de estrategias de formación modernas, como lo demuestran Hernández-Ramos, et al. (2014) y Paechter y Maier (2010). Por ello, es importante que las nuevas demandas académicas consideren patrones que pautan un enfoque moderno de la educación, ya que, el auto-aprendizaje y la autonomía son dos condiciones que motivan al estudiante $\mathrm{y}$, por ende, inciden en su comportamiento. Lo anterior se corrobora con la disposición positiva que tienen los estudiantes hacia a la innovación pedagógica y didáctica, y cómo esto incide en su motivación por conocer y probar nuevas tecnologías de la información y las comunicaciones en diferentes ámbitos y contextos de su vida.

Los hallazgos para cada una de las variables consideradas en los modelos de aceptación tecnológica dejan abierta la posibilidad de evaluar la interdependencia entre los factores del modelo, de modo que se pueda verificar el poder explicativo de factores determinantes como la facilidad de uso y la utilidad percibida, a partir de las variables que están determinadas por las percepciones que tienen los estudiantes acerca de las herramientas virtuales y de las capacidades de los docentes que los incentivan a utilizarlas, y con ello, desarrollar estrategias de seguimiento y evaluación en la implementación de este tipo de estrategias pedagógicas y didácticas para la medicación del proceso de enseñanza-aprendizaje. Por lo anterior, los resultados evidencian que los esfuerzos que el gobierno nacional, mediante el Plan Nacional de Tecnologías de la Información y las Comunicaciones 20082019, ha adelantado para que se incentive la utilización de tecnologías de la información $y$ las comunicaciones en instituciones educativas, como estrategia de masificación y modernización de los modelos educativos en su oferta académica (Ministerio de Telecomunicaciones, 2008) son positivos.

Finalmente, aunque los resultados obtenidos refuerzan el conocimiento respecto a la incidencia que tiene el uso de plataformas virtuales en los estudiantes en relación con su utilidad y facilidad de uso, no son concluyentes para determinar los efectos que las estrategias de e-learning están produciendo en la 
percepción que tienen los estudiantes respecto a la calidad de los programas académicos de Educación Superior, lo que deja abierta la discusión para futuras investigaciones en este mismo campo.

\section{Referencias bibliográficas}

- Artino, A. y Jones, K. (2012). Exploring the complex relations between achievement emotions and self-regulated learning behaviors in online learning. The Internet and Higher Education, 15(3), 170-175.

- Artino, A. (2010). Online or face-to-face learning? Exploring the personal factors that predict students' choice of instructional format. The Internet and Higher Education, 13(4), 272-276.

- Benta, D.; Bologa, G. y Dzitac, L. (2014). E-learning platforms in higher education. case study. Procedia Computer Science, 31, 1170-1176.

- Chen, J. (2011). The effects of education compatibility and technological expectancy on e-learning acceptance. Computers \& Education, 57(2), 1501-1511.

- Domingo, M. y Garganté, A. (2016). Exploring the use of educational technology in primary education: Teachers' perception of mobile technology learning impacts and applications' use in the classroom. Computers in Human Behavior, 56, 21-28.

- El-Seoud, S.; Taj-Eddin, I.; Seddiek, N.; ElKhouly, M. y Nosseir, A. (2014). E-Learning and Students' Motivation: A Research Study on the Effect of E-Learning on Higher Education. iJET, 9(4), 20-26.

- Essalmi, F.; Ben Ayed, L.; Jemni, M. y Graf, S. (2010). A fully personalization strategy of E-learning scenarios. Computers in Human Behavior, 26(4), 581-59.

- Fragoso, P. y Perechona, P. (2013). Didáctica de la educación en valores en la ESO. Una propuesta utilizando las tecnologías para el aprendizaje y el conocimiento. Pixel-Bit, 42, 195 - 208.

- Garrison, D. (2011). E-learning in the 21st century: A framework for research and practice. New York: Taylor \& Francis.

- González, N.; García, R. y Ramírez, A. (2015). Aprendizaje cooperativo y tutoría entre iguales en entornos virtuales universitarios. Estudios pedagógicos (Valdivia), 41(1), 111-124.
- Hamidi, F.; Meshkat, M.; Rezaee, M. y Jafari, M. (2011). Information technology in education. Procedia Computer Science, 3, 369373.

- Hernández-Ramos, J.; Martínez-Abad, F.; García, F.; Herrera, E. y Rodríguez-Conde, M. (2014). Teachers' attitude regarding the use of ICT. A factor reliability and validity study. Computers in Human Behavior, 34, 509-516.

- Hung, E.; Valencia, J. y Silveira, A. (2016). Factores determinantes del aprovechamiento de las TIC en docentes de educación básica en Brasil. Un estudio de caso". Perfiles Educativos, XXXVIII (151), 71-85.

- Klašnja-Milićevića, A.; Vesina, B.; Ivanovićb, M. y Budimacb, Z. (2011). E-Learning personalization based on hybrid recommendation strategy and learning style identification. Computers \& Education, 56(3), 885-899.

- Lee, L. y Hung, J. (2015). Effects of blended e-Learning: a case study in higher education tax learning setting. Human-centric Computing and Information Sciences, 5(1), 1-15.

- Lu, H. (2012). Learning styles and acceptance of e-learning management systems: an extension of behaviour intention model". International Journal of Mobile Learning and Organisation, 6(3-4), 246-259.

- Mcgill, T.; Klobas, J. y Renzi, S. (2014). Critical success factors for the continuation of e-learning initiatives. The Internet and Higher Education, 22, 24-36.

- Ministerio de Telecomunicaciones. (2008). Plan Nacional de Tecnologías de la información y las comunicaciones. Bogotá D. C.: Ministerio de Telecomunicaciones.

- Mohammadi, H. (2015). Investigating users' perspectives on e-learning: An integration of TAM and IS success model. Computers in Human Behavior, 45, 359-374.

- Mohammadyari, S. y Singh, H. (2015). Understanding the effect of e-learning on individual performance: The role of digital literacy. Computers \& Education, 82, 11-25.

- Norris, P. (2001). Digital divide: Civic engagement, information poverty, and the Internet worldwide. Cambridge: Cambridge University Press.

- Oviedo, H. y Campo-Arias, A. (2005). Aproximación al uso del coeficiente alfa de Cronbach. Revista colombiana de psiquiatría, 34(4), 572-580.

- Ozdamli, F. y Uzunboylu, H. (2015). M-learning adequacy and perceptions of students and 
teachers in secondary schools. British Journal of Educational Technology, 46 (1), 159-172.

- $\quad$ Paechter, M. y Maier, B. (2010). Online or faceto-face? Students' experiences and preferences in e-learning. The internet and higher education, 13(4), 292-297.

- Park, Y. y Lim, K. (2015). Effects of Environmental and Human Constructs on E-Learning Effectiveness in Online University Settings. Indian Journal of Science and Technology, 8(S1), 103-109.

- $\quad$ Park, S. (2009). An analysis of the technology acceptance model in understanding university students' behavioral intention to use e-learning. Journal of Educational Technology \& Society, 12(3), 150-162.

- Rosenberg, M. (2001). E-learning: Strategies for delivering knowledge in the digital age. New York: McGraw-Hill.
- Sánchez, R. y Hueros, A. (2010). Motivational factors that influence the acceptance of Moodle using TAM. Computers in human behavior, 26(6), 1632-1640.

- Tarhini, A.; Hassouna, M.; Abbasi, M. y Orozco, J.. (2015). Towards the Acceptance of RSS to Support Learning: An empirical study to validate the Technology Acceptance Model in Lebanon. Electronic Journal of e-Learning, 13(1), 30-41.

- Unesco. (2005). Hacia las sociedades del conocimiento. París: Ediciones Unesco.

- Zhang, X.; Wang, W.; De Pablos, P.; Tang, J. y Yan, X. (2015). Mapping development of social media research through different disciplines: collaborative learning in management and computer science. Computers in Human Behavior, 51b, 1142-1153. 